\title{
LA REALIDAD VIRTUAL EN LAS INTERVENCIONES PSICOLÓGICAS CON PACIENTES ONCOLÓGICOS
}

\section{PSYCHOLOGICAL INTERVENTION USING VIRTUAL REALITY FOR PATIENTS WITH CANCER}

\author{
Macarena Espinoza', Rosa M. Baños ${ }^{2}$, Azucena García-Palacios ${ }^{1,3}$ y Cristina Botella \\ Universitat Jaume I, Castellón \\ 2 Universidad de Valencia \\ ${ }^{3}$ CIBER Fisiopatología Obesidad y Nutrición (CBO6/03), Instituto de Salud Carlos III, Madrid
}

Resumen

El objetivo de este trabajo es presentar las principales aportaciones de la Realidad Virtual (RV) en el tratamiento psicológico con pacientes oncológicos, y describir un programa de intervención que utiliza esta tecnología para promover bienestar emocional en pacientes hospitalizados. La RV se presenta como una prometedora herramienta de apoyo al paciente oncológico. Diversos estudios plantean su utilidad para hacer algunos tratamientos médicos más tolerables, para reducir los niveles de distrés y dolor, así como promover mayores niveles de emociones positivas. Se ha empleado principalmente con pacientes ambulatorios, existiendo una menor proporción de propuestas dirigidas al período de hospitalización. La intervención que ha desarrollado nuestro grupo aborda esta condición desde un foco también poco explorado: la promoción de bienestar. Esta intervención se compone de 4 sesiones (módulos de distracción y reminiscencia) implementadas individualmente en la habitación del paciente. Antes y después de cada sesión se evaluó el estado emocional (escalas visuales), la percepción de paso del tiempo (en minutos), de cambio en el estado de ánimo y de satisfacción con el procedimiento. Los resultados iniciales señalan incrementos en las emociones positivas después de cada sesión, existiendo cambios significativos en la primera
Abstract

The purpose of the present study is to review the main contributions of Virtual Reality (VR) to the field of psychological treatment with cancer patients, and to describe an intervention that uses this technology to promote emotional wellbeing on inpatients. VR appears as a promising tool for supporting psychological treatments in cancer patients. Several studies explore its utility for promoting more tolerable medical treatments, lowering the level of distress and pain, as well as increasing positive emotions. VR has been mainly used with outpatients, with a minor proportion of proposals oriented to the hospitalization period. The intervention developed by our group addresses this issue from a focus not very much explored: the promotion of well-being. This intervention is composed of 4 sessions (distraction and reminiscence modules) implemented individually in the patients' room. Emotional state (visual analog scales), perceived time duration (minutes), perception of mood change and satisfaction with the procedure were assessed before and after sessions. Preliminary results showed an increase in positive emotions after each session, being these changes significant in the first and third session. Besides, most patients perceived themselves in a better mood after the sessions.

Virtual environments and information and communication technologies could be useful

\section{Correspondencia:}


y tercera sesión. Además, la mayoría de pacientes informó que su estado de ánimo había mejorado después de las sesiones.

A partir de estos hallazgos se observa que los entornos virtuales y las tecnologías de la información y la comunicación muestran utilidad no sólo para disminuir el malestar sino también para promover bienestar emocional, especialmente durante períodos de tratamiento y hospitalización.

Palabras Clave: Realidad virtual, cáncer, distrés, bienestar emocional.

\section{INTRODUCCIÓN}

En los últimos años, las Tecnologías de la Información y la Comunicación (TICs) están ofreciendo muchas ventajas y potencialidades en la administración de los tratamientos psicológicos en general, y en el campo oncológico en particular. El uso de Internet, por ejemplo, ha posibilitado que los programas terapéuticos puedan Ilegar a aquellos pacientes que les resulta difícil el traslado hasta sus centros de salud, ya sea por la enfermedad, la ubicación geográfica o cualquier otra razón ${ }^{(1-3)}$. Otro ejemplo lo constituyen los denominados "juegos serios" (serious games), juegos digitales creados con propósitos que van más allá del entretenimiento, que facilitan la entrega de información médica y de herramientas de apoyo para la toma decisiones y la implicación en conductas saludables ${ }^{(4-6)}$. En los últimos años se está también comenzando a indagar acerca de la viabilidad y los beneficios asociados a la evaluación electrónica en este tipo de pacientes, fundamentalmente mediante ordenador ${ }^{(7-9)}$, y se han generado aplicaciones móviles dirigidas a monitorizar y ayudar al manejo de la sintomatología desde casa(10-11), además de servir de apoyo a programas online, gracias a la posibilidad que ofrecen para registrar síntomas, recibir mensajes y acceder a módulos del programa ${ }^{(12)}$. Si bien estos desarrollos son todavía escasos, se están not only to diminish discomfort but also to promote emotional well-being, especially during medical procedures and hospitalization

Key words: Virtual reality, cancer, distress, emotional well-being. obteniendo resultados preliminares alentadores, lo que sugiere su potencialidad en términos de evaluación e intervención en este grupo de pacientes.

Una de las TICs que se ha utilizado en el ámbito oncológico es la Realidad Virtual (RV). La RV es una tecnología que permite crear ambientes tridimensionales en los que es posible interactuar con cualquier objeto. En ese sentido, Burdea $(1993)^{(13)}$ definió la RV como "una compleja interfaz de usuario que engloba simulaciones e interacciones en tiempo real a través de múltiples canales sensoriales. Estas modalidades sensoriales son visuales, auditivas, táctiles, olfativas, etc.". ¿Qué ha Ilevado a que los investigadores apuesten por la utilidad de la RV en el campo de las intervenciones psicológicas? La RV ofrece una serie de ventajas importantes ${ }^{(14)}$. Además de su versatilidad y la posibilidad de modelar cualquier mundo o cualquier situación, la RV favorece la emergencia de emociones, sensaciones, pensamientos y reacciones similares a las que el paciente experimentaría ante eventos reales semejantes. Lo que pretende la RV es que el usuario se perciba "dentro" de esa realidad y no ajeno a ella, lo que da pie para trabajar con vivencias que puedan ser significativas para el individuo ${ }^{(15)}$. En ese sentido, la RV ofrece un "ambiente seguro" donde explorar a un ritmo personalizado, dando la posibilidad de experimentarse a sí mismo y al mundo 
de un modo alternativo. Además, la RV es flexible y por tanto adaptable a las necesidades del individuo (situaciones, grado de dificultad, etc.), dando al terapeuta la posibilidad de ofrecer al paciente contextos y situaciones que le sean significativas y resulten de relevancia para su problemática, todo lo cual puede ser de gran ayuda para el proceso terapéutico ${ }^{(15)}$.

El presente trabajo se centrará en las principales aportaciones de la RV al campo de las intervenciones psicológicas en pacientes oncológicos. Además de resumir los principales hallazgos y usos que se han dado a la RV en el campo de la Psico-Oncología, un segundo objetivo se centra en describir la propuesta de intervención que se encuentra actualmente en desarrollo en nuestro país, y que utiliza RV junto con otras TICs, y cuya finalidad es la promoción de bienestar emocional en pacientes oncológicos hospitalizados.

\section{Realidad Virtual y Psico-Oncología}

En la actualidad ya contamos con varios estudios que han utilizado la RV como herramienta útil para el manejo de los síntomas asociados al proceso de enfermedad oncológica $y$, en particular, de aquellos vinculados a los procedimientos médicos. Estos estudios se han dirigido mayoritariamente hacia dos focos: por un lado, evaluar el impacto de la RV como herramienta terapéutica orientada al manejo del dolor y la ansiedad ante procedimientos médicos dolorosos, y por otro, indagar en las posibilidades que ofrece la RV para el manejo de síntomas de distrés (ansiedad, fatiga) durante el proceso de quimioterapia.

La primera de estas áreas de investigación se ha apoyado en gran medida en el potencial distractor que posee la RV y en la utilidad que ello tiene a la hora de disminuir la percepción de dolor de los pacientes. Estos estudios, en su mayoría, han tenido como grupo objetivo a niños y adolescentes con cáncer que deben someterse a procedimientos como inyecciones, punciones o implantaciones de catéteres. En ellos se ha constatado que la RV favorece la disminución del dolor y ansiedad durante los procedimientos ${ }^{(16-18)}$ así como el miedo existente ${ }^{(19)}$, siendo considerada por los pacientes como una experiencia positiva para ellos ${ }^{(20)}$.

En relación con la segunda gran área de estudio en pacientes con cáncer, se han utilizado intervenciones basadas en RV durante las sesiones de quimioterapia, con la finalidad de alterar la percepción del paso del tiempo de los pacientes, de modo que el tratamiento se experimente como más breve y se vivencie mejor, resultando con ello un procedimiento mucho más tolerable ${ }^{(21,22)}$. A este respecto, Schneider, Kisby y Flint $(2011)^{(23)}$ han observado que el diagnóstico es una variable relevante, encontrando que es más probable que los pacientes con cáncer de mama y colon experimenten una mayor percepción alterada del tiempo durante las sesiones con RV. Según estos autores, tal resultado podría deberse a la menor presencia de sintomatología en pacientes con estos diagnósticos, ya que los datos señalan que un nivel elevado de sintomatología hace que la atención se dirija en mayor proporción hacia los síntomas que se estan experimentando, inhibiendo la capacidad del paciente para "sumergirse" en los ambientes virtuales.

Otros estudios han investigado la utilidad de las intervenciones basadas en RV durante la aplicación de la quimioterapia para reducir los niveles de ansiedad ${ }^{(24)}$, distrés y fatiga ${ }^{25)}$. Del mismo modo, se ha propuesto que la intervención con RV podría ser de utilidad para disminuir no sólo la fatiga y el estado emocional negativo, sino también las náuseas y vómitos experimentados durante las sesiones de quimioterapia ${ }^{(26)}$ así como los vómitos posteriores a este tratamiento en pacientes adultos ${ }^{(27)}$. Con respecto a esto último, sin embargo, 
existen resultados contradictorios, ya que un estudio con mayor tamaño muestral(21) ha señalado que la intervención con RV no implica una mejora en los síntomas asociados al procedimiento de quimioterapia. Este estudio además no ha detectado diferencias significativas en los niveles de distrés experimentados por los pacientes.

Además, no sólo se han investigado los efectos de los tratamientos basados en RV sobre los efectos inmediatos tras la quimioterapia, sino que también se ha identificado una tendencia hacia la mejora en los síntomas de ansiedad, fatiga y distrés, durante las 48 horas posteriores ${ }^{(24)}$. Sin embargo, es preciso contar con una mayor cantidad de estudios que estimen la extensión y capacidad de mantenimiento de los efectos alcanzados, en vista de que hay algunos datos que señalan la ausencia de efectos duraderos en las mejorías obtenidas inmediatamente después de las sesiones con quimioterapia ${ }^{(28)}$ y puesto que los datos existentes se han obtenido con muestras de diferentes características.

Además de estas dos grandes áreas de trabajo, se han propuesto también otras aplicaciones de la RV en el ámbito del cáncer, intentando abordar otros aspectos de relevancia en el proceso de enfermedad, como son la entrega de información y el manejo del estrés y las preocupaciones acerca del cáncer durante la hospitalización. Por ejemplo, Greene (1998) ${ }^{(29)}$ sugirió el empleo de la RV como plataforma para realizar un programa educativo que abordara tres grandes áreas: cáncer de mama, dolor y prevención. Para ello se incorporó no sólo información médica, sino también elementos de la historia personal, con el fin de favorecer una aproximación individual a los contenidos en vez de que el paciente interactúe con situaciones abstractas. Este programa se encuentra disponible en la página Web de la Universidad de Michigan (CommTechLab, http://commtechlab.msu. edu/products/index.html). Este mismo gru- po ha desarrollado un programa psicoeducativo interactivo para pacientes con enfermedad avanzada y al final de la vida, que ha sido valorado positivamente por los pacientes (mayoritariamente ambulatorios) en términos de agrado y facilidad de uso $^{(30)}$. Sin embargo, no se han efectuado estudios para valorar la efectividad de estas propuestas.

Otra área en la que también se han desarrollado intervenciones basadas en RV ha sido durante la hospitalización. Por ejemplo, Oyama, Wakao y Okamura $\left(1997^{(31)}\right.$ sugirieron emplear la RV dentro de un programa para disminuir el estrés y las preocupaciones durante el período de hospitalización, destinando esta tecnología para la presentación de películas personales, cartas de parte de familiares y amigos, instrucciones personales acerca de exámenes médicos e información sobre la enfermedad. Con esta propuesta se obtuvieron buenos resultados preliminares que indicaban disminuciones en los niveles de estrés, si bien es preciso señalar que no ha sido evaluada en posteriores estudios. Este mismo grupo ${ }^{(32)}$ desarrolló además una intervención con RV orientada a la promoción de mayores niveles de relajación y de estados emocionales positivos. Este sistema, denominado "Sistema de Bienestar al lado de la cama", incluye paseos por un bosque virtual, donde el paciente puede experimentar efectos de sonido, viento y aromas. Los autores señalaron como resultado preliminar, un aumento de las emociones positivas y una disminución de las negativas en un grupo de mujeres con cáncer de mama y ovario. Este programa de intervención se amplió, existiendo tres ambientes virtuales disponibles para el paciente (bosque, lago y pueblo) así como la posibilidad de que el individuo escogiera entre varias esencias aromáticas y el formato de audio que deseaba (altavoces o audífonos). Sin embargo, este programa ampliado se ha evaluado sólo como herramienta de manejo de síntomas asociados a quimioterapia $^{(27)}$. 
Un estudio más reciente efectuado en China, ha empleado un espacio virtual interactivo con el propósito de implementar una intervención de juego para niños hospitalizados. La intervención consistió en 5 sesiones grupales (con un máximo de 4 niños) de media hora cada una, durante una semana. Una vez finalizada la intervención, se detectó una disminución significativa en la sintomatología depresiva de aquellos niños que participaron de la intervención con el espacio virtual ${ }^{(33)}$.

A partir de lo anteriormente expuesto se constata que gran parte de los estudios realizados en el ámbito del cáncer se han orientado a pacientes ambulatorios y a la reducción de síntomas, existiendo, de momento, una menor proporción de estudios dirigidos a la situación de hospitalización y al fomento de bienestar emocional en estos pacientes. Resulta importante continuar en esta línea, ya no sólo intentando aminorar el malestar sino también fomentando los recursos personales y el bienestar psicológico de estos pacientes. Los positivos resultados obtenidos hasta el momento sugieren que la incorporación de este tipo de tecnologías puede ser una ayuda valiosa durante el proceso de enfermedad, especialmente en contextos de tratamiento y hospitalización.

El ambiente hospitalario conlleva una serie de exigencias y experiencias que pueden ser vividas como estresantes: es un ambiente donde el individuo debe dejar sus roles habituales, asumir un rol más dependiente y afrontar lo incierto ${ }^{(34)}$. En ese sentido, se ha planteado que el ambiente hospitalario fuerza al individuo a ceder el control sobre su vida diaria ${ }^{(35)}$ y se vuelve un espacio donde emerge incertidumbre respecto de los síntomas, tratamiento y resultado de éste ${ }^{(36)}$. Asimismo, la hospitalización supone restricciones en cuanto a movilidad y estimulación, pudiendo afectar a la propia sensación de competencia.
A partir de todo esto se entiende que estar hospitalizado no es sólo una situación vinculada al proceso de enfermedad, sino que además puede llegar a experimentarse como una fuente de múltiples situaciones estresantes, capaces de impactar sobre necesidades fundamentales como la autonomía, la competencia y la posibilidad de relación con otros.

Considerando lo anterior, se aprecia la utilidad de generar espacios alternativos dentro de este contexto, en los cuales los pacientes tengan la posibilidad de reforzar sus propios recursos, desarrollar nuevos, y experimentar sensaciones agradables y de bienestar. Las intervenciones positivas se centran en este objetivo, dado que se han encontrado beneficios en calidad de vida, morbilidad y bienestar psicológico asociados al hecho de ejercitar las emociones positivas y fortalezas personales ${ }^{(37-40)}$. Efectuar intervenciones centradas en la promoción del bienestar con pacientes ingresados, resulta por tanto un intento de refuerzo personal en un contexto de malestar, con el propósito de aportar experiencias que amplíen los recursos disponibles del paciente para afrontar el período de ingreso hospitalario.

\section{UNA INTERVENCIÓN PSICOLÓGICA APOYADA EN TICS ORIENTADA A LA PROMOCIÓN DE BIENESTAR EMOCIONAL}

Nuestro grupo de investigación ha desarrollado una intervención basada en RV, cuya finalidad es la promoción de bienestar emocional de pacientes oncológicos adultos hospitalizados. Su objetivo es ofrecer un espacio de distracción y/o tranquilidad dentro de la rutina hospitalaria, estimular el desarrollo de imágenes mentales asociadas a sensaciones agradables y favorecer el recuerdo de momentos positivos y significativos de la propia historia. A continuación vamos a ofrecer una 
descripción del programa, junto con datos preliminares de su eficacia, ya que se trata de un estudio aún en curso.

La intervención está compuesta por dos módulos: distracción y reminiscencia, que se aplican a lo largo de 4 sesiones ( 2 centradas en distracción y 2 en reminiscencia).

El módulo de distracción incluye dos entornos virtuales (Parque y Paseo por la Naturaleza) (ver la figura 1), en los que el paciente tiene la posibilidad de realizar diversas actividades encaminadas a promover y ejercitar estados emocionales positivos (alegría, relajación), a la vez que genera una imagen mental del entorno y sus características. El objetivo, por tanto, es que el paciente no sólo obtenga beneficios inmediatos de la interacción en los ambientes virtuales (distracción, entretenimiento, tranquilidad) sino que, además, pueda emplear la representación mental de estos ambientes como un recurso a utilizar en momentos de malestar emocional (lugar mental al cual acudir en momentos de tensión, ansiedad, etc.).

Los entornos cuentan con características especialmente diseñadas para promover las emociones positivas de alegría y relax (música, colorido, sonidos, iluminación, elementos de la naturaleza), junto con una serie de actividades orientadas a tal propósito (trabajo con frases tipo Velten, imágenes, fragmentos de películas, recuerdo autobiográfico, respiración lenta, atención plena a los estímulos del entorno). Una descripción más detallada de ambos entornos virtuales se puede encontrar en trabajos previos ${ }^{(41-43)}$ (se puede acceder a las imágenes incluidas en presente artículo en: http://www.labpsitec.uji.es/esp/ multimedia/imagenesONC.php)

El módulo de reminiscencia incluye una herramienta multimedia denominada "Libro de la Vida", que permite al paciente explorar experiencias pasadas, y registrar pensamientos, sentimientos y vivencias relacionadas con cada situación en una especie de diario personal (ver la figura 2). El sistema cuenta con 7 temas de la propia historia que es posible revisar: uno de ellos apunta a una revisión de vida en general, y los otros 6 temas exploran momentos positivos (momento de felicidad, de logros y esfuerzo personal, de valor, recibiendo afecto de otros, disfrute de las relaciones sociales y agradecimiento).

En la intervención propuesta se sugiere a los pacientes explorar cuatro de estos momentos positivos (momento de felicidad, de logros, disfrute de las relaciones sociales y agradecimiento). Cada temática es abordada mediante una serie de preguntas que permiten profundizar en el recuerdo y sus detalles. Los distintos momentos a explorar se presentan al paciente como capítulos de un libro que irán elaborándose a lo largo de las sesiones. El sistema permite escribir así como incorporar diversos elementos multimedia a cada uno de los capítulos del libro

\section{Figura 1. Entornos Virtuales}

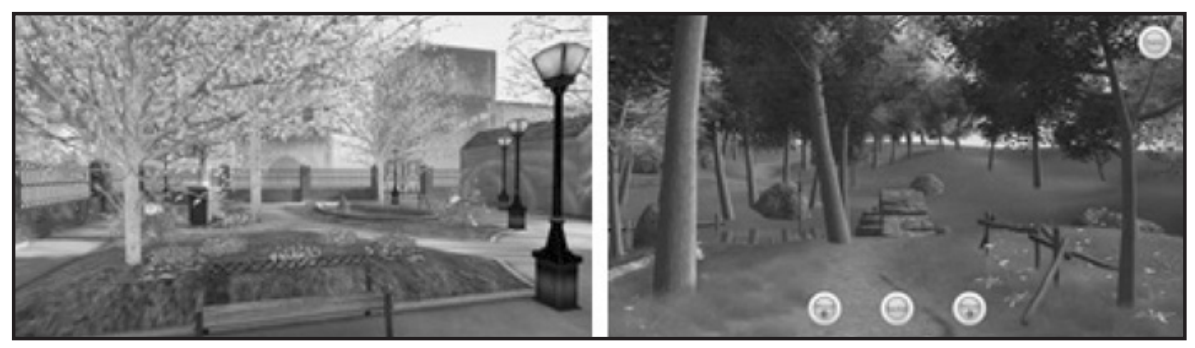


personal (imágenes, música y videos), con el fin de potenciar la evocación y elaboración del recuerdo. Este material multimedia puede ser personalizado.

Como antes señalábamos, la intervención completa se compone de 4 sesiones llevadas a cabo de forma individual durante 1 semana, en la habitación del paciente. Dos de estas sesiones están orientadas al módulo de reminiscencia (1era y 3era sesión) y dos al módulo de distracción (2da y 4ta sesión). En el caso del módulo de reminiscencia, se propone revisar 2 momentos por sesión, definidos previamente. En el módulo de distracción, los pacientes pueden escoger el entorno que desean visitar en cada sesión. Después de cada una de las sesiones se sugiere el recuerdo de lo trabajado en sesión.

Para presentar la intervención se utiliza una televisión LCD de 32 pulgadas conec- tada a un ordenador, todo dispuesto en un soporte móvil que permite el desplazamiento de una habitación a otra dentro del hospital (figura 3). Se emplean audífonos para el sonido (para el paciente y terapeuta), el teclado del ordenador para redactar los capítulos del Libro de la Vida, y se emplea un Rumblepad y un TrackBall como dispositivos de interacción y desplazamiento en los entornos virtuales (ver la figura 3).

Con el propósito de reforzar los contenidos vistos en sesión, así como de conocer el estado emocional y físico de los pacientes en el período entre-sesiones, se han desarrollado además dos aplicaciones para móvil (ver las figuras 4 y 5). Este sistema de apoyo a la intervención ha sido diseñado para móviles con sistema operativo Android. Sus características se describen a en la tabla 1.

\section{Figura 2. Sistema Libro de la Vida}

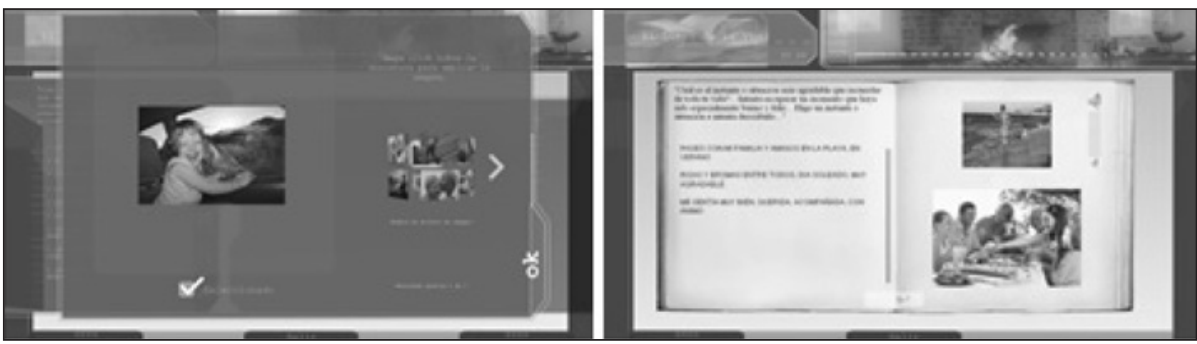

Figura 3. Aparatos Intervención mediante TICs

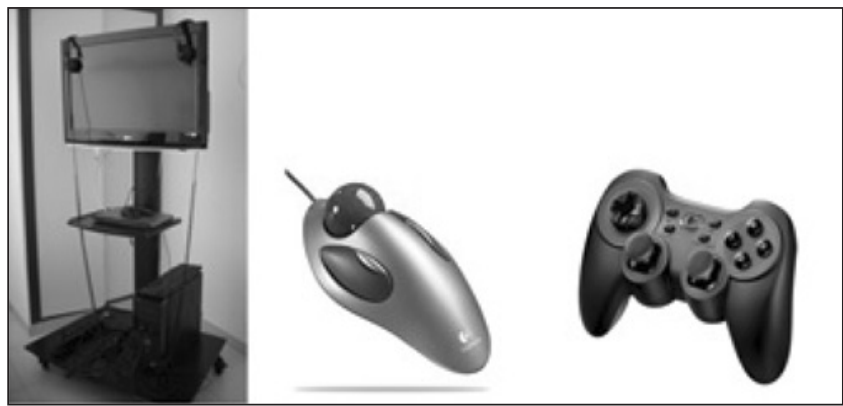




\section{Tabla 1. Apps de Apoyo a la Intervención}

\begin{tabular}{|c|c|}
\hline \multicolumn{2}{|c|}{ Características Generales } \\
\hline App Intervención & App Evaluación \\
\hline $\begin{array}{l}\text { Módulo Distracción: Contiene } 5 \text { videos breves } \\
\text { de recorridos por los entornos virtuales (Parque } \\
\text { Alegre, Parque Relax, Árbol de Tranquilidad, } \\
\text { Bienestar junto al río, Paseo al Lago) }\end{array}$ & $\begin{array}{l}\text { Permite el registro diario del estado de ánimo y } \\
\text { estado físico del paciente. } \\
\text { Las variables que explora son: afecto positivo, } \\
\text { negativo, fatiga, energía, náuseas y malestar físico. }\end{array}$ \\
\hline $\begin{array}{l}\text { Módulo Reminiscencia: Listado de } 7 \text { temas para } \\
\text { recordar. En cada uno de ellos existe un par de } \\
\text { preguntas que facilitan el recuerdo. }\end{array}$ & $\begin{array}{l}\text { Se utilizan escalas visuales para indicar las } \\
\text { respuestas. Cada pregunta se presenta también } \\
\text { en formato audio. }\end{array}$ \\
\hline \multicolumn{2}{|c|}{ Modo de Empleo } \\
\hline App Intervención & App Evaluación \\
\hline $\begin{array}{l}\text { Módulo Distracción: El paciente puede } \\
\text { seleccionar los videos que desee ver. Las } \\
\text { opciones disponibles son activadas por el } \\
\text { terapeuta en función de lo trabajado en las } \\
\text { sesiones presenciales. }\end{array}$ & $\begin{array}{l}\text { La evaluación se activa una vez al día, en un } \\
\text { horario acordado previamente con el paciente. } \\
\text { Una alarma indica al paciente que debe } \\
\text { contestar una serie de preguntas. }\end{array}$ \\
\hline $\begin{array}{l}\text { Módulo Reminiscencia: El paciente selecciona } \\
\text { aquellos temas que desea revisar. Tiene la } \\
\text { opción de redactar frases significativas que se } \\
\text { almacenan para su posterior lectura. Los temas } \\
\text { disponibles son activados por el terapeuta. }\end{array}$ & $\begin{array}{l}\text { El terapeuta selecciona las variables que desea } \\
\text { evaluar en cada paciente, y establece el período } \\
\text { de tiempo durante el cual se mantendrá activa } \\
\text { esta evaluación. }\end{array}$ \\
\hline
\end{tabular}

Figura 4. App Intervención

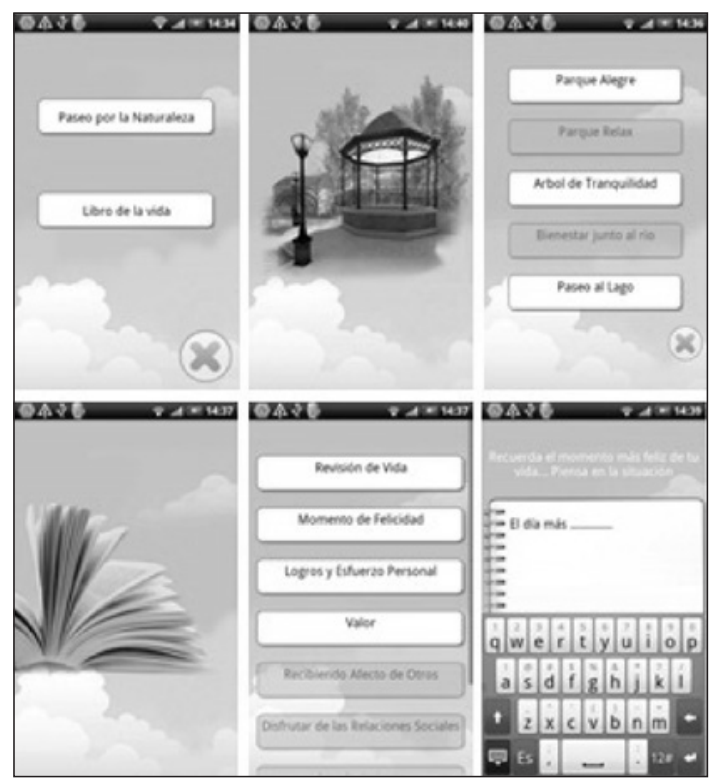




\section{Figura 5. App Evaluación}

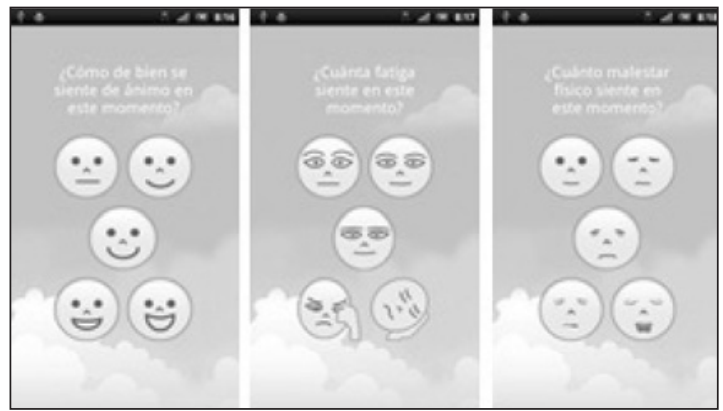

\section{RESULTADOS PRELIMINARES}

Actualmente se está llevando a cabo un estudio clínico controlado con el propósito de valorar la eficacia y eficiencia de esta propuesta de intervención. Debido a que aún no existen datos suficientes para comparar los resultados del grupo de pacientes que ha recibido la intervención con aquellos que se encuentran en la condición control (tratamiento médico habitual), se presentan los resultados de 11 pacientes que han realizado una o más sesiones. De momento no contamos con datos de las aplicaciones móviles, por lo que no es posible presentar resultados de tales desarrollos.

\section{Características de los participantes}

Los pacientes incluidos son adultos con cualquier tipo de cáncer que se encuentren hospitalizados al menos durante 1 semana, que presenten un estadio funcional de Karnofsky $\geq 50$, tengan una expectativa de vida $\geq 2$ meses en el momento de la inclusión, y que no presenten trastornos psicopatológicos graves o alteraciones cognitivas. Todos los pacientes procedían de la planta de oncología del Hospital Clínica Benidorm.

La media de edad de los participantes fue de 58,7 años (rango 38-82 años), la mayoría eran mujeres $(63,6 \%)$, casadas o que convivían con su pareja $(63,6 \%)$, con nivel bajo de estudios (54,5\%) (esto es, sabe leer y escribir=9,1\%; nivel bási$\mathrm{co}=45,5 \%)$ y jubiladas $(54,5 \%)$. El principal diagnóstico fue "otro" (linfoma, cáncer retroperitoneal, cáncer de laringe) $(27,3 \%)$ y cáncer de pulmón (18,2\%). La mayoría presentaba cáncer metastásico $(63,6 \%)$ y tenían un estado funcional de Karnofsky medio de $82(\mathrm{DT}=6,5)$.

\section{MEDIDAS}

\section{Escalas Visuales Analógicas}

Se valoraron cuatro variables: estado de ánimo positivo, estado de ánimo negativo, bienestar y tranquilidad en el momento actual. Cada uno de ellos fue puntuado en una escala que va de 0 (nada) a 4 (mucho). Las escalas de ánimo positivo y negativo están representadas por símbolos faciales y las de bienestar y tranquilidad son escalas visuales numéricas.

\section{Evaluación Subjetiva de Cambio en Estado de ánimo}

Se realizó una pregunta para estimar la presencia de cambios en el estado de ánimo entre antes y después de cada sesión de intervención, empleándose como criterio de comparación el estado anímico 
que el sujeto presentaba antes de iniciar la sesión. Para ello, se le indicó que ubicase su respuesta en una escala de 0 a 4 que iba de "Peor" a "Mejor".

\section{Percepción Tiempo}

Se solicitó a los pacientes que indicasen, en minutos, la cantidad de tiempo que había durado cada sesión. Además de este valor subjetivo, se registró la duración real de cada sesión.

\section{Satisfacción con el procedimiento}

Al finalizar cada sesión se estimó el nivel de agrado y utilidad percibida de la sesión, utilizando una escala que va de 0 (nada) a 4 (mucho).

\section{PROCEDIMIENTO}

Todas las sesiones fueron administradas de forma individual en la habitación de los pacientes. Antes de comenzar, se les explicaba la estructura y contenidos del programa, indicándoles la existencia de dos sistemas con los que era posible trabajar: entornos virtuales y libro de la vida. Se les aclaraba el modo de funcionamiento del sistema y se daba paso a la sesión. La 1era y 3era sesión correspondían a sesiones del módulo de reminiscencia y la $2 \mathrm{da}$ y 4ta sesión al módulo de distracción. La terapeuta se encontraba presente durante todo el procedimiento. Antes y después de cada sesión se realizó una evaluación del estado emocional de los pacientes, de su percepción de paso del tiempo y grado de satisfacción con el procedimiento.

\section{RESULTADOS}

\section{Resultados Pre-Post Sesión}

Al analizar el cambio en las medidas emocionales después de cada una de las sesiones realizadas, se comprueba que en todas ellas hubo un incremento en las medidas emocionales positivas. Específicamente, en la 1era (ánimo positivo, $p=0,020$, $\mathrm{Z}=-2,333, \mathrm{~d}=-0,68$; bienestar, $p=0,059$, $\mathrm{Z}=-1,890, \mathrm{~d}=-0,67)(\mathrm{n}=11)$ y 3era sesión (ánimo positivo, $p=0,034, \mathrm{Z}=-2,121, \mathrm{~d}=$ 1,20 ; bienestar, $p=0,039, Z=-2,060, d=-$ 1,63; tranquilidad, $p=0,034, \quad Z=-2,121$, $\mathrm{d}=-1,44)(\mathrm{n}=8)$ estos cambios alcanzaron significación estadística con tamaños del efecto aceptables (entre moderados y grandes). En la 1era sesión se observó además un cambio relevante en tranquilidad $(p=0,083 ; Z=-1,732 ; d=-0,47)$, si bien no alcanzó a ser estadísticamente significativo. Para llevar a cabo estos análisis se empleó la prueba de Wilcoxon.

Asimismo, se comprobó que la duración percibida de cada sesión fue menor a la duración real. Esta diferencia fue estadísticamente significativa en la 1era sesión, con un tamaño del efecto grande ( $\mathrm{Z}=-2,135 ; p=0,033 ; \mathrm{d}=-1,08)$. Esto apunta no sólo al potencial distractor de las sesiones, sino que puede considerarse a su vez como una medida de bienestar.

Por último, con respecto a la percepción de cambio en el estado de ánimo después de finalizadas las sesiones, se observó que en todas ellas la mayor parte de los pacientes (en todas las sesiones por encima del 60\%) se encontró "mejor" de ánimo (ver Gráfico 1). Destacan aquí las dos sesiones dirigidas al trabajo con reminiscencia (1era y 3era sesión), que son las que a su vez obtuvieron mejorías estadísticamente significativas en los niveles de bienestar emocional.

\section{Satisfacción con las Sesiones}

Los pacientes valoraron de forma positiva cada una de las sesiones (escala 0-4). La sesión mejor evaluada fue la 3era, que corresponde al módulo de reminiscencia (ver la tabla 2). 


\section{Gráfico 1. Evaluación Subjetiva de Cambio en Estado de ánimo}

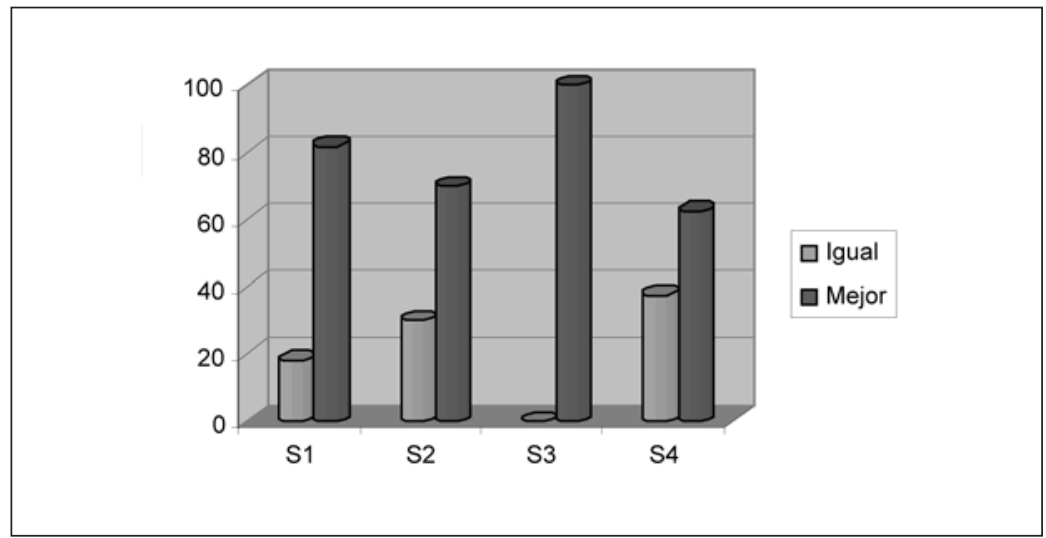

Tabla 2. Niveles de Agrado y Utilidad percibida Sesiones

\begin{tabular}{|l|c|c|c|c|c|c|c|c|}
\hline & \multicolumn{2}{|c|}{ Sesión 1 } & \multicolumn{2}{c|}{ Sesión 2 } & \multicolumn{2}{c|}{ Sesión 3 } & \multicolumn{2}{c|}{ Sesión 4 } \\
\hline & $\mathrm{X}$ & $\mathrm{DT}$ & $\mathrm{X}$ & $\mathrm{DT}$ & $\mathrm{X}$ & $\mathrm{DT}$ & $\mathrm{X}$ & $\mathrm{DT}$ \\
\hline Agrado & 3,05 &, 65 & 3,10 &, 74 & 3,56 &, 50 & 3,25 &, 71 \\
\hline Utilidad & 2,73 &, 79 & 2,40 & 1,43 & 3,13 &, 83 & 2,88 & 1,25 \\
\hline
\end{tabular}

\section{CONCLUSIONES}

El foco de la mayoría de los trabajos con RV en el ámbito oncológico ha estado principalmente puesto en reducir el malestar derivado de los tratamientos médicos, fundamentalmente en reducir la ansiedad, fatiga, dolor y vómitos asociados a la quimioterapia. Los datos recabados hasta el momento indican que las intervenciones basadas en RV son eficaces para disminuir el dolor y ansiedad, para promover una experiencia más agradable durante las sesiones de quimioterapia, y para disminuir el malestar posterior a la quimioterapia. Sin embargo, aún no es posible determinar la potencia que tiene la disminución del malestar post quimioterapia, ni el efecto que tiene una intervención con RV en los vómitos asociados a este tratamiento.
Por otro lado, se constata la escasez de intervenciones basadas en RV diseñadas para facilitar la situación de hospitalización en pacientes oncológicos. Hasta ahora se ha intentado favorecer la disminución del estrés y las preocupaciones, focalizándose para ello en la entrega de información y la creación de nexos que favorezcan el contacto con otros significativos. Se han desarrollado, además, sistemas que buscan fomentar el estado emocional positivo en este contexto. Atendiendo a los alentadores resultados que se han obtenido hasta ahora con los sistemas de RV en el campo oncológico, parece importante continuar investigando en esta línea, especialmente por lo que se refiere a la potencialidad de esta herramienta para la promoción de mayores niveles de bienestar en el contexto hospitalario. Si bien existen estudios 
que han abordado el fomento del bienestar emocional en pacientes ingresados, de acuerdo con nuestro conocimiento, la mayor parte de estos trabajos son propuestas de intervención que no cuentan con datos de eficacia, lo que plantea la necesidad de continuar con la evaluación y validación de éstas y otras herramientas de apoyo al bienestar.

En este trabajo hemos descrito el programa de intervención desarrollado por nuestro grupo. La experiencia que se ha conseguido hasta el momento con el sistema aquí descrito sugiere la viabilidad de su implementación en el contexto hospitalario, especialmente si se atiende a la brevedad de las sesiones propuestas, ya que ello facilita el ajuste a los horarios y exigencias de la rutina hospitalaria ${ }^{(44,43)}$. Además, las sesiones se realizan en la misma habitación del paciente, posibilitando con ello que aquellos pacientes que no puedan desplazarse con facilidad, también tengan acceso a esta intervención.

Si bien de momento la muestra ha sido reducida, los resultados iniciales indican la presencia de mejorías en los niveles de bienestar después de cada una de las sesiones realizadas, lo que respalda la intervención propuesta. Es de especial interés el hecho de que las sesiones con mayor percepción de mejoría y las que alcanzaron cambios estadísticamente significativos en bienestar, son las dos sesiones dedicadas al trabajo con reminiscencia. Puede pensarse que este trabajo personal de revisión de momentos significativos positivos ha sido capaz de aportar no sólo una conexión con emociones positivas y de bienestar (como el módulo de distracción), sino que además ha permitido incorporar aspectos personales significativos relacionados con la propia identidad, un elemento que podría estar detrás de los buenos resultados obtenidos con este módulo y que será preciso continuar explorando con un mayor número de pacientes.
Aún cuando de momento no existen datos de la utilidad de las aplicaciones móviles, pensamos que éstas pueden resultar de gran apoyo para el período entre sesiones o para cuando el paciente ya se encuentre en casa. Estas aplicaciones ofrecen la posibilidad de reforzar experiencias vinculadas con sensaciones agradables y permiten ejercitar el recuerdo de vivencias personales positivas, todo lo cual puede servir como "un respiro" dentro del contexto hospitalario, así como un recurso más del cual poder disponer ante futuras situaciones difíciles. El hecho de que estas aplicaciones incorporen además una evaluación diaria del estado del paciente, permite un conocimiento más próximo de las fluctuaciones a nivel emocional y del estado físico, lo que resulta muy relevante si se desea ofrecer apoyo acorde con las necesidades del paciente.

A partir de la revisión efectuada y la experiencia en curso, puede plantearse que las TICs son útiles para promover mayores niveles de bienestar y menores niveles de malestar en distintos grupos de pacientes oncológicos (niños, adolescentes y adultos) y en distintos contextos (ambulatorios y hospitalizados). Es de especial interés continuar explorando las ventajas y oportunidades que este tipo de tecnologías pueden ofrecer al trabajo terapéutico en particular, y a los profesionales de la salud en general, con el fin de ampliar los recursos de los que se dispone actualmente para dar respuesta a las demandas de los pacientes y familiares de pacientes con cáncer. En ese sentido, puede ser de utilidad rescatar las ventajas que ofrece la RV para el trabajo terapéutico, especialmente en contextos poco tradicionales como el del ingreso hospitalario. La RV da la posibilidad de "trasladar" al paciente a entornos y situaciones que puedan resultar más confortables y menos amenazantes, generando un espacio en el que sea factible retomar el control personal. De este modo, 
se entiende que la RV y las TICs no sólo estarían facilitando la distracción respecto del entorno hospitalario, sino que además posibilitarían al paciente nuevas experiencias de aprendizaje y de vivencia emocional, lo que puede resultar de gran apoyo para intervenciones que desean promover bienestar en situaciones difíciles.

\section{REFERENCIAS BIBLIOGRÁFICAS}

1. Chambers S, Girgis A, Occhipinti S, Hutchison S, Turner J, Carter R, et al. Beating the blues after Cancer: Randomised controlled trial of a tele-based psychological intervention for high distress patients and carers. BMC Cancer 2009;9:189. Doi:10.1186/1471-2407-9-189

2. Owen J, Klapow J, Roth D, Shuster J, Bellis J, Meredith R, et al. Randomized pilot of a self-guided internet coping group for women with early-stage breast cancer. Ann Behav Med 2005;30:54-64.

3. Shepherd L, Goldstein D, Whitford H, Thewes B, Brummell V, Hicks M. The utility of videoconferencing to provide innovative delivery of psychological treatment for rural cancer patients: Results of a pilot study. J Pain Symptom Manag 2006;32:453-61.

4. Kato P, Cole S, Bradlyn A, Pollock B. A video game improves behavioral outcomes in adolescents and young adults with cancer: A randomized trial. Pediatrics 2008;122:e305-e317. Doi:10.1542/ peds.2007-31345.

5. Reichlin L, Mani N, McArthur K, Harris A, Rajan N, Dacso C. Assessing the acceptability and usability of an interactive serious game in aiding treatment decisions for patients with localized prostate cancer. J Med Internet Res 2011;13:246-60.

6. Roubidoux M. Breast Cancer Detective: A computer game to teach breast cancer screening to native american patients. J Cancer Educ 2005;20:S1:87-91.

7. Carlson L, Speca M, Hagen N, Taenzer P.Computerized quality-of-life screening in a cancer pain clinic. J Palliat Care 2001;17:46-52.

8. Chan C, Tam W, Cheng K, Chui Y, So W, Mok $\mathrm{T}$, et al. Piloting electronic self report symptom assessment e Cancer (ESRA-C) in Hong Kong: A mixed method approach. Eur J Oncol Nurs 2011;15:325-34.

9. Thekkunpurath $\mathrm{P}$, Venkateswaran C, Kumar M, Newsham A, Bennett M. Screening for psychological distress in palliative care: Performance of touch screen questionnaires compared with semistructured psychiatric interview. J Pain Symptom Manag 2009;38:597-605. Doi:10.1016/j. jpainsymman.2009.01.004

10. Kearney N, Kidd L, Miller M, Sage M, Khorrami J, McGee $M$, et al. Utilising handheld computers to monitor and support patients receiving chemotherapy: Results of a UK-based feasibility study. Support Care Cancer 2005; Doi:10.1007/s00520005-0002-9

11. McCann L, Maguire M, Kearney N. Patients' perceptions and experiences of using a mobile phone-based advanced symptom management system (ASyMSC) to monitor and manage chemotherapy related toxicity. Eur J Cancer Care 2009;18:156-64.

12. Mirkovic J, Bryhni H, Ruland C. Designing User Friendly Mobile Application to Assist Cancer Patients in Illness Management. eTELEMED 2011: The Third International Conference on eHealth, Telemedicine, and Social Medicine 2011; 64-71.

13. Burdea G. Virtual Reality Systems and Applications. Electro'93 International Conference, Short Course. Edison, NJ; april 28; 1993.

14. Botella C, García-Palacios A, Quero S, Baños R, Bretón-López J. Realidad virtual y tratamientos psicológicos: Una revisión. Psicol Conduct 2006;3:491-510.

15. Botella C, Quero S, Baños RM, Perpiñá C, García-Palacios A, Riva G. Virtual Reality and Psychotherapy. En: Riva G, Botella C, Légeron P, Optale G, editores. Cybertherapy: Internet and virtual reality 
as assessment and rehabilitation tools for clinical psychology and neuroscience. Amsterdam: IOS Press, 2004.

16. Gershon J, Zimand E, Lemos R, Rothbaum B, Hodges L. Use of virtual reality as a distractor for painful procedures in a patient with pediatric cancer: A case study. Cyberpsychol Behav 2003;6:657-61.

17. Gershon J, Zimand E, Pickering M, Rothbaum B, Hodges L. A Pilot and feasibility study of virtual reality as a distraction for children with cancer. J Am Acad Child Psy 2004;43:1243-9.

18. Wolitsky K, Fivush R, Zimand E, Hodges $L$, Rothbaum B. Effectiveness of virtual reality distraction during a painful medical procedure in pediatric oncology patients. Psychol Health 2005;20:817-24.

19. Windich-Biermeier A, Sjoberg I, Dale J, Eshelman D, Guzzetta C. Effects of distraction on pain, fear, and distress during venous port access and venipuncture in children and adolescents with cancer. J Pediatr Oncol Nurs 2007;24: 8-19.

20. Nilsson S, Finnstrôm B, Kokinsky E, Enskâr $\mathrm{K}$. The use of virtual reality for needle-related procedural pain and distress in children and adolescents in a paediatric oncology unit. Eur J Oncol Nurs 2009;13:102-9.

21. Schneider S, Hood L. Virtual Reality: A distraction intervention for chemotherapy. Oncol Nurs Forum 2007;34:39-46.

22. Schneider S, Workman M. Virtual reality as a distraction intervention for older children receiving chemotherapy. Pediatr Nurs 2000;26:593-7.

23. Schneider S, Kisby C, Flint E. Effect of virtual reality on time perception in patients receiving chemotherapy. Support Care Cancer 2011;19:555-64. Doi: 10.1007/ s00520-010-0852-7

24. Schneider S, Ellis M, Coombs W, Shonkwiler $\mathrm{E}$, Folsom L. Virtual reality intervention for older women with breast cancer. Cyberpsychol Behav 2003;6:301-7.

25. Schneider S, Prince-Paul M, Allen M, Silverman $\mathrm{P}$, Talaba D. Virtual reality as a distraction intervention for women receiving chemotherapy. Oncol Nurs Forum 2004;31:81-8.

26. Kaneda M, Oyama H, Katsumata N. VR Intervention therapy for emotion related cancer chemotherapy side effects. Int Conf Artif Real Telexistence 1999;9:98-101.

27. Oyama H, Kaneda M, Katsumata N, Akechi T, Oshuga M. Using the bedside wellness system during chemotherapy decreases fatigue and emesis in cancer patients. J Med Syst 2000;24(3):173-182.

28. Schneider S, Workman M. Effects of virtual reality on symptom distress in children receiving chemotherapy. CyberPsychol Behav 1999;2:125-134.

29. Greene D. Personal Stories within Virtual Environments: Creating three experiences in cancer information software. En: Riva, G., Widerlhold, B. y Molinari E., editors. Virtual Environment in Clinical Pychology and Neuroscience. Amsterdam: IOS Press, 1998.

30. Lambing A, Markey C, Neslund-Dudas C, Bricker L. Completing a Life: Comfort level and ease of use of a CD-ROM among seriously III patients. Oncol Nurs Forum 2006;33:999-1006.

31. Oyama H, Wakao F, Okamura H. Virtual reality support system in palliative medicine. St Heal T 1997;39:60-63.

32. Oyama H, Oshuga M, Tatsuno Y, Katsumata N. Evaluation of the psycho-oncological effectiveness of the bedside wellness system. Cyberpsychol Behav 1999;2(1):8184.

33. Li W, Chung J, Ho E, Chiu S. Effectiveness and feasibility of using the computerized interactive virtual space in reducing depressive symptoms of Hong Kong Chinese children hospitalized with cancer. J Spec Pediatr Nurs 2011;16:190-198. Doi: 10.1111/j.1744-6155.2011.00288.x

34. Koenig H, George L, Stangl D, Tweed D. Hospital stressors experienced by elderly medical inpatients: developing a hospital stress index. Int J Psychiat Med 1995;25:103-22. 
35. Taylor S. Hospital patient behavior: Reactance, helplessness, or control?. J Soc Issues 1979;35:156-84.

36. Merle M. Perceived uncertainty and stress in illness. Res Nurs Health 1984;7:163-71.

37. Cohn M, Fredrickson, B. In search of durable positive psychology interventions: Predictors and consequences of long-term positive behavior change. J Posit Psychol 2010;5:355-66.

38. Folkman S, Moskowitz J. Positive affect and the other side of coping. Am Psychol 2000;55:647-54

39. Lyubomirsky S, King L, Diener E. The benefits of frequent positive affect: does happiness lead to success? Psychol Bull 2005;131:803-55.

40. Seligman M, Rashid T, Parks A. Positive psychotherapy. Am Psychol 2006;774-88.

41. Baños RM, Botella C, Rubio I, Quero S, García-Palacios A, Alcañiz M. Presence and emotions in virtual environment: The influence of estereoscopy. CyberPsychol Behav 2008;11:1-8.

42. Botella C, Etchemendy E, Castilla D, Baños RM, García-Palacios A, Quero S, et al. An ehealth system for the elderly (butler project): A pilot study on acceptance and satisfaction. Cyberpsychol Behav 2009;12:255-262.

43. Espinoza $M$, Baños RM, García-Palacios A, Alcañiz M, Botella C, Cervera JM, et al. Descripción de un sistema de realidad virtual dirigido a la promoción de bienestar emocional en pacientes oncológicos hospitalizados: ensayo fase II OncoHelp. Rev Int Grupos Inv Oncol 2012;1:40-6.

44. Baños RM, Espinoza M, García-Palacios A, Cervera JM, Esquerdo G, Barrajón E, et al. A positive psychological intervention using virtual reality for patients with advanced cancer in a hospital setting: a pilot study to assess feasibility. Support Care Cancer 2013;21:263-70. Doi:10.1007/ s00520-012-1520-x 
